\title{
Thanks to reviewers
}

We greatly appreciate the voluntary contribution that each reviewer gives to the Journal. We hope that our reviewers will help us by reviewing the articles submitted to BIBECHANA in future also.

Prof. Devendra Adhikari

Editor-in-Chief

BIBECHANA

http://nepjol.info/index.php/BIBECHANA/index

\section{Reviewers}

Dr. Alev Dogan, Gazi University, Ankara 6100, Turkey.

Dr. Xilian Jin, College of Physics, Jilin University, China.

Dr. Mohd Nazri Idris, Universiti Sains Malaysia, Engineering Campus, Malaysia.

Dr. Aditya Mahabhai Vora, B. V. Shah Science College, Gujarat, India.

Dr. B.P. Singh, T.M. Bhagalpur University, Bhagalpur, India.

Dr. Nicola Seriani, ICTP, Strada Costeera 11, 34151 Trieste, Italy.

Prof. Ramazan Sever, Middle East Technical University, Turkey.

Dr. Martin Lopez Corredira, Instituto de Astrofísica de Canarias, Tenerife, Spain.

Prof. R.N. Joarder, Jadavpur University, Kolkata-700 032, India.

Dr. G.S. Shivaprasanna, Amruta institute of Eng. and Manag. Sc., Bangalore.

Dr. B.C. Paul, University of North Bengal, India.

Prof. Md. Haider Biswas, Khulna University, Khulna-9208, Bangladesh.

Prof. R.B. Chaudhary, BRA, Bihar University, Muzafffarpur, India.

Prof. Badiadka Narayana, Mangalore University, India.

Prof. B.K. Singh, T.M. Bhagalpur University, Bhagalpur.

Dr. Basant Giri, University of Wyoming, Wyoming, USA. 